\title{
Small Deletion at the 7q21.2 Locus in a CCM Family Detected by Real-Time Quantitative PCR
}

\author{
Lucia Anna Muscarella, ${ }^{1}$ Vito Guarnieri, ${ }^{2}$ Michelina Coco, ${ }^{1,2}$ Serena Belli, ${ }^{3}$ Paola Parrella, ${ }^{1}$ \\ Giuseppe Pulcrano, ${ }^{4}$ Domenico Catapano, ${ }^{5}$ Vincenzo A. D'Angelo, ${ }^{5}$ Leopoldo Zelante, ${ }^{2}$ \\ and Leonardo D'Agruma²
}

${ }^{1}$ Laboratory of Oncology, IRCCS “Casa Sollievo della Sofferenza” Hospital, 71013 San Giovanni Rotondo (FG), Italy
${ }^{2}$ Medical Genetics Unit, Evolutive Age Department, IRCCS “Casa Sollievo della Sofferenza” Hospital,
71013 San Giovanni Rotondo (FG), Italy
${ }^{3}$ Medical Genetics Department, APSS Trento, 38123 Trento, Italy
${ }^{4}$ Neurosurgical Operative Unit, "Santa Chiara” Hospital, 38123 Trento, Italy
${ }^{5}$ Neurosurgical Operative Unit, IRCCS “Casa Sollievo della Sofferenza” Hospital, 71013 San Giovanni Rotondo (FG), Italy

Correspondence should be addressed to Lucia Anna Muscarella, 1.muscarella@operapadrepio.it

Received 31 March 2010; Accepted 24 June 2010

Academic Editor: Meena Upadhyaya

Copyright (C) 2010 Lucia Anna Muscarella et al. This is an open access article distributed under the Creative Commons Attribution License, which permits unrestricted use, distribution, and reproduction in any medium, provided the original work is properly cited.

\begin{abstract}
Cerebral cavernous malformations (CCMs) represent a common autosomal dominant disorder that predisposes patients to haemorrhagic strokes and focal neurological signs. About $56 \%$ of the hereditary forms of CCMs have been so far associated with mutations in the KRIT1 (Krev Interaction Trapped 1) gene, located at 7q21.2 (CCM1 locus). We described the complete loss of 7q21.2 locus encompassing the KRIT1 gene and 4 flanking genes in a CCM family by using a dense set of 12 microsatellite markers. The complete loss of the maternal copy of KRIT1 gene region was confirmed by Real-Time Quantitative Polymerase Chain Reaction (RT-QPCR) and the same approach was used for expression analysis. Additional RT-QPCR analysis showed the extension of the deletion, for a total of $700 \mathrm{~kb}$, to the adjacent downstream and upstream-located genes, MTERF, AKAP9, CYP51A1, as well as a partial loss of the ANKIB1 gene. Here we report the molecular characterization of an interstitial small genomic deletion of the 7q21.2 region in a CCMs affected family, encompassing the KRIT1 gene. Our findings confirm the loss of function mechanism for the already known CCM1 locus, without any evident involvement of the other deleted genes. Moreover, our investigations highlight the usefulness of the RT-QPCR to the molecular characterization of the breakpoints genomic deletions and to the identification of internal deleted genes involved in the human genetic diseases.
\end{abstract}

\section{Introduction}

Cerebral cavernous malformations (CCMs, [MIM 116860]) are congenital vascular defects mostly located within the CNS with a prevalence of $0.1 \%-0.5 \%$ in the general population [1]. They are characterized by abnormally enlarged capillary cavities without intervening brain parenchyma [2]. The presence of these altered vascular structures is believed to account for all symptoms, ranging from headache to focal neurological defects and rarely acute bleeding. CCMs are also associated to an increased probability of stroke and epilepsy $[1,3,4]$. Family cases are often characterized by the presence of multiple lesions with an incomplete penetrance probably related to gene involved $[5,6]$ and on the age at onset. Heritable CCMs have been so far associated with mutations in three genes: the KRIT1 (Krev Interaction Trapped 1) [7] and the CCM2 (Cerebral Cavernous Malformation 2, Malcavernin) genes located, respectively, at the 7q21.2 (CCM1 locus) and 7p15-p13 (CCM2 locus) [8], and the PDCD10 (Programmed Cell Death 10) gene is located at the 3q26.1-27 (CCM3 locus) [9]. KRIT1 gene is responsible for about $56 \%$ of the hereditary forms of CCMs, whereas the MGC4607 gene accounted for 33\% of them. The third locus identified by mutational screening of the PDCD10 gene 
surprisingly showed mutations only in a low percentage of familial case $(6 \%)$, suggesting the existence of a fourth gene located close to one of the three loci above mentioned $[9,10]$.

Recently, several papers reported that many probands initially negative at the routine mutation screening for the three CCM genes were positive for large genomic deletions or duplications. Deletion of malcavernin seems to be the most frequent genomic rearrangement reported in CCMs families whereas one duplication, 9 partial deletions, and 1 deletion of the whole KRIT1 gene were described [11-15].

Here we describe for the first time the molecular characterization of the complete loss of CCM1 genomic region in a CCMs family, by using a combination of microsatellite markers analysis and an RT-QPCR approach. Other than the KRIT1, four genes fall in the deleted region: MTERF (mitochondrial transcription termination factor), AKAP9 (A-kinase-anchoring protein), CYP51A1 (cytochrome P450, family 51), and ANKIB1 (ankyrin repeat and IBR domain containing 1) gene whose first 10 exons were deleted. These results were corroborated by gene expression analysis by using RT-QPCR.

\section{Patients and Methods}

2.1. Patients. All the subjects belonged to a Northern Italian family affected by cerebral cavernous malformation. The index case is a 36-year-old male with a seizure history and cerebral haemorrhages. The five family members were investigated by brain TC and MRI and the diagnosis of CCM was based on its characteristic radiographic findings. Each subject underwent detailed clinical assessment, with emphasis on neurological, dermatological, and ophthalmological examinations. Informed consent was obtained from all family members to perform genetic analyses.

2.2. Microsatellite Analysis. DNA was extracted from peripheral blood using a standard phenol-chloroform protocol [16]. Linkage analysis was performed in order to identify the disease associated gene. Haplotypes analyses were performed for all CCMs family members by using a dense set of 13 microsatellite markers flanking the CCM1 locus (See Table 1 in Supplementary Material available online at doi:10.1155/2010/854737). Physical distances between markers were based upon the electronic database available from the University of California Santa Cruz (http://www.genome.ucsc.edu/). Amplifications were carried out in 25 ul reaction volume containing $100 \mathrm{ng}$ of DNA, 10X PCR Buffer with $15 \mathrm{mM} \mathrm{MgCl}_{2}, 200 \mu \mathrm{M}$ each dNTPs, 20 pmol each primer, and $1 \mathrm{U}$ AmpliTaq Gold DNA polymerase (Applied Biosystems, Foster City, CA). PCR cycling conditions consisted of initial 12 minutes denaturation step at $95^{\circ} \mathrm{C}$, followed by 35 cycles of $95^{\circ} \mathrm{C}$ for 30 seconds, annealing for 30 seconds, and extension at $72^{\circ} \mathrm{C}$ for 30 seconds, with final extension at $72^{\circ} \mathrm{C}$ for 7 minutes. PCR products were visualised by ethidium bromide staining on $2 \%$ agarose gel. PCR products were loaded on a capillary electrophoresis ABI 3100 (Applied Biosystems), and results were analysed by using Genescan 3.7 and Genotyper 3.7 NT software (Applied Biosystems).
2.3. KRIT1 Gene Screening. The mutational analysis of the KRIT1 gene was performed as previously described [17]. Briefly, amplifications for all the 16 KRIT1 coding regions, including the exon-intron boundaries, were carried out and analysed by denaturing high-performance liquid chromatography (DHPLC; Transgenomic Inc. Transgenomic, Inc. Nebraska, USA) screening (primers sequences, annealing temperatures, and size of PCR products are in Supplementary Table 1; oven temperatures and acetonitrile gradients are available from the authors). Amplicons with an abnormal elution profile were purified using the GFX PCR and Band Purification Kit (Ge HealthCare, Buckinghamshire, UK), sequenced with the BigDye Terminator Cycle Sequencing Kit v. 1.1 (Applied Biosystems), loaded on ABI 3100 capillaries (Applied Biosystems) and analysed using the Sequencing Analysis software v2.0.

2.4. RT-QPCR Gene Copy Number Analysis. A new protocol of RT-QPCR was developed to provide a sensitive method for detecting large deletions encompassing the KRIT1 gene and the $7 \mathrm{q} 21$ locus.

Three sets of primers were designed for amplification of 7 q21 region around the KRIT1 gene (Supplementary Table 2). All primers were designed with Primer Express 2.0 software (Applied Biosystems) and tested for specificity using BLAT software (http://genome.ucsc.edu/cgi_bin/ hgBlat?command=start). Primer sets 1 identify 8 amplicons covering the KRIT1 gene (exons 3,9,17, and 20) and $5^{\prime}$ and $3^{\prime}$ flanking $4.5 \mathrm{~Kb}$ regions. Primer set 2 annealed on the 10 KRIT1 flanking genes located into the $7 q 21$ region delimited by D7S2410 and D7S646 microsatellites: PFTK1 (NM_012395), FZD1 (NM_003505), MTERF (NM_006980), AKAP9 (NM_005751), CYP51A1 (NM_000786), ANKB1 (NM_019004.1), GATAD1 (NM_021167), ERVWE1 (NM_ 014590), PEX1 (NM_000466), and CDK6 (NM_001259).

The identification of ANKIB1 gene breakpoint region was performed using an additional set of primers (set 3 ) designed on exons $7-13$ of the gene (Supplementary Table 3). All primers were purchased by PRIMM (PRIMM Labs, Inc. MI, Italy).

Deletion of KRIT1 gene and of the flanking genes was detected on the 384-well ABI Prism 7900 Sequence Detection System (Applied Biosystems) by the measurements of the amplicons Copy Number (CN) using a RT-QPCR approach with SYBR-Green I detection. Reaction mixture (10 ul) contained 2.5x Fast Start DNA master mix hybridization SYBR Green (Roche Molecular Biochemicals, Mannheim, Germany), $250 \mathrm{uM}$ of each forward and reverse primers, and $30 \mathrm{ng}$ of DNA as template. Reactions were run on ABI PRISM 7900HT Sequence Detection System (Applied Biosystems). Cycling conditions were as follows: 10 minutes at $95^{\circ} \mathrm{C}, 40$ cycles at $95^{\circ} \mathrm{C}$ for 15 seconds, and $60^{\circ} \mathrm{C}$ for 60 seconds. After PCR amplification, a melting curve was generated for every PCR product in order to verify the specificity of the PCR reaction. Calculation of the gene copy number was made using the $2^{-\Delta \Delta C T}$ method as reported (User Bulletin \#2, Applied Biosystems) [18, 19]. Outlier values with a differences between $\mathrm{Ct}$ and $\mathrm{Ct}$ mean $>0.3$ were excluded from further data analysis. For normalization of the 


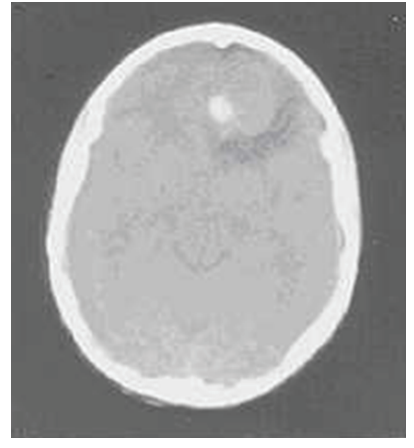

FIgURE 1: Unenhanced CT image of the index case (Case II:2) showing a large lesion with a hyperattenuating centre surrounded by little spots of increased density (suggestive for calcifications and small areas of haemorrhage) in the left frontal lobe. Large edema coexists. Further histological examination proved this lesion to be a CCM.

relative amount, the gene copy numbers were divided by the geometric mean of two described reference with a normal copy number, ZNF80 (3q13.31) and MOX2 (3q13.2) [20]. Using this method, a Diploid Copy Number (D-CN) of 1.0 \pm 0.2 is expected for a normal sample and a value of $0.5 \pm$ 0.2 for a sample with 7q21 genomic deletion (Haploid Copy Number, H-CN).

\subsection{Sample Processing, RNA Extraction and cDNA Synthesis.} Total RNA of I : $1, \mathrm{I}: 2$, II $: 1$, and II $: 3$ subjects was extracted using PAXgene Blood RNA kit (PreAnalytix, Qiagen, Germantown, $\mathrm{MD}$ ). RNA was eluted in RNAse free-water and stored at $-80^{\circ} \mathrm{C}$ until used. RNA quality and concentration were measured by using 2100 Expert Analyzer (Agilent Technology, Inc. Headquarters) with an RIN (RNA Integrity Number) $\geq 9.0$. After heating at $65^{\circ} \mathrm{C}$ for 5 minutes in order to denature RNA and to inactivate RNases, $500 \mathrm{ng}$ of total RNA was subjected to reverse transcription using the QuantiTect Reverse Transcription Kit (Qiagen). cDNA was synthesized according to the manufacturer's instructions.

2.6. RT-QPCR Gene Expression Analysis. Primers set were designed with Primer Express 2.0 software (Applied Biosystems) across the coding region of flanking KRIT1 genes into the 7q21 deleted region: PFTK1, FZD1, MTERF, AKAP9, CYP51A1, KRIT1, ANKB1, GATAD1, ERVWE1, PEX1, and CDK6 (Supplementary Table 4).

SYBR Green amplification mixture (10 ul) contained 2.5x QuantiTect SYBR Green PCR Master Mix (Qiagen), $250 \mathrm{nM}$ of each forward and reverse primer, and $1 \mathrm{ul}$ of cDNA as template. Reactions run on ABI PRISM 7900HT Sequence Detection System (Applied Biosystems). Cycling conditions were as follows: 10 minutes at $95^{\circ} \mathrm{C}, 40$ cycles at $95^{\circ} \mathrm{C}$ for 15 seconds, and $60^{\circ} \mathrm{C}$ for 60 seconds. Glyceraldehyde phosphate dehydrogenase $(G A P D H)$ was chosen as housekeeping gene, and commercially available primers were used (see the User Bulletin \#2, Applied Biosystems, for the primers sequences). Each assay was carried out in triplicate and the transcription level was normalized using GAPDH as reference gene.

\section{Results}

3.1. Clinical Descriptions. The index case (Case II:2) is a 36 years old male. When he was 11 years old, he had an episode of severe headache and language disturbances. CT examination performed at that moment showed a haemorrhagic lesion in the left frontal lobe (Figure 1). Therefore, he underwent surgical treatment with good results. MRI followup examination was performed every two years, the last of which revealed two lesions suggestive for hemangiomas, respectively, located at the cerebellopontine angle and at the left lateral ventriculum. The mother of the index case (Case $\mathrm{I}: 2)$ is a 61 years old female. When she was 48 years old, an MRI examination of the orbit performed after an episode of right blindness associated to acute headache showed an area of haemorrhage at the superior right quadrant and at the inferior left quadrant of the right eye. She underwent surgical treatment. At present, a visual defect at the right eye still persists. The brother of the index case (Case II : 1) is a 38 years old male with a history of recurrent headaches and seizures due to multiple cerebral hemorrhages from the age of 9. Surgical treatment was performed on a cerebral hemangioma located at the right parietal lobe. He underwent MRI follow-up examinations every two years. The last one revealed three lesions suggestive for hemangiomas: 1 in the right fronto parietal lobe, 1 at the cerebellopontine angle, and 1 in the cerebellum. The nephew of the index case (Case III : 1) is an 11 years old male. When he was 5 years old, he reported a seizure. MRI showed two cerebral lesions suggestive for hemangiomas, one of them was bleeding. Only the father of the index case (Case I:1) showed negative results at MRI and CT examinations. In the three patients (Cases I :2, II : 1, II :2) who underwent surgical treatment, the diagnosis of CCM was histologically confirmed. To date, all the family members are alive.

\subsection{Genetic Analysis}

\subsubsection{Identification of Hemizigosity at the 7q21 Chromosome} Region. Haplotype reconstruction of 13 microsatellite markers on chromosome 7q21 showed heterozygosity for markers flanking the CCM1 locus (D7S2409, D7S1813, D7S1789) in the healthy family member $(\mathrm{I}: 1)$, whereas all the affected members (I:2, II $: 1$, II $: 3$, III $: 1)$ carried and shared only the maternal haplotype, indicating a hemizygosity of the specific chromosomal region of about $700 \mathrm{~kb}$ (Figure 2(a)). The genetic screening of the whole coding regions of the KRIT1 gene gave negative results.

3.2.2. Identification of Complete KRIT1 and Flanking Genes Deletions by RT-QPCR. By using the RT-QPCR approach with the three primer sets described above we confirmed the complete deletion of KRIT1 gene and the hemizygous status in all affected patients (Supplementary Figure 1). Moreover, the deletion of the $5^{\prime}$ and $3^{\prime}$ KRIT1 flanking region was found involving the following genes: MTERF, AKAP9, CYP51A1, located downstream to the KRIT1 gene, and ANKIB1 located upstream to the KRIT1 gene (Figure 2(b)). 


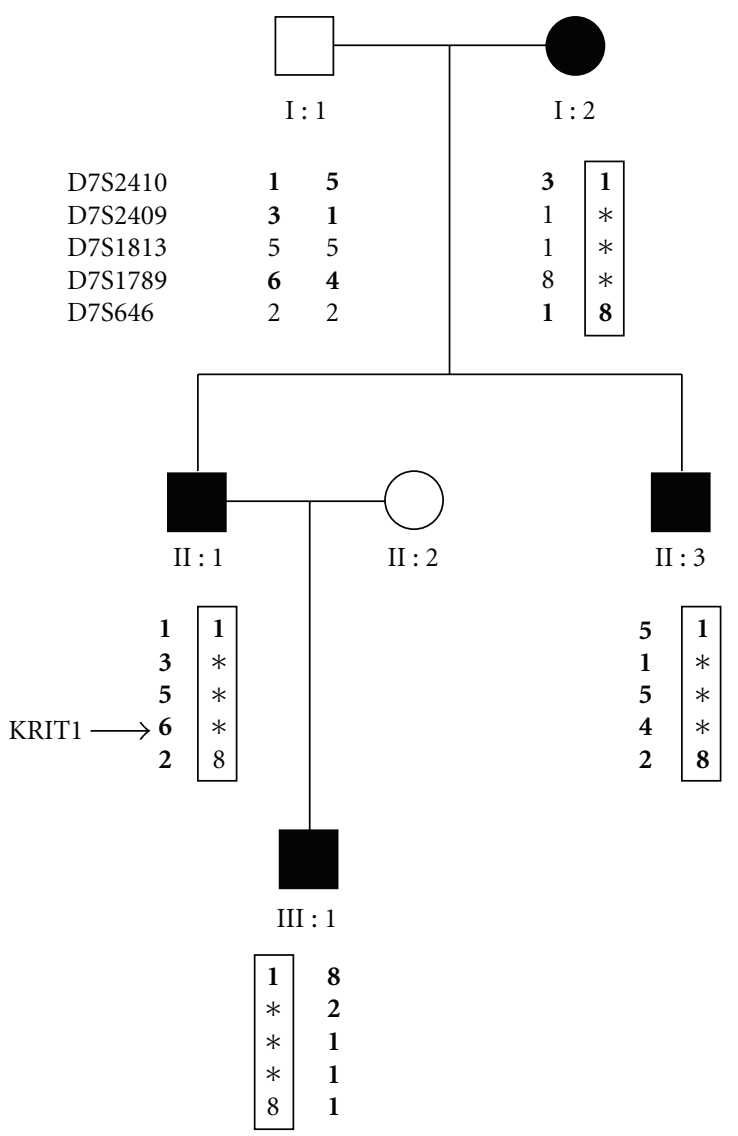

(a)
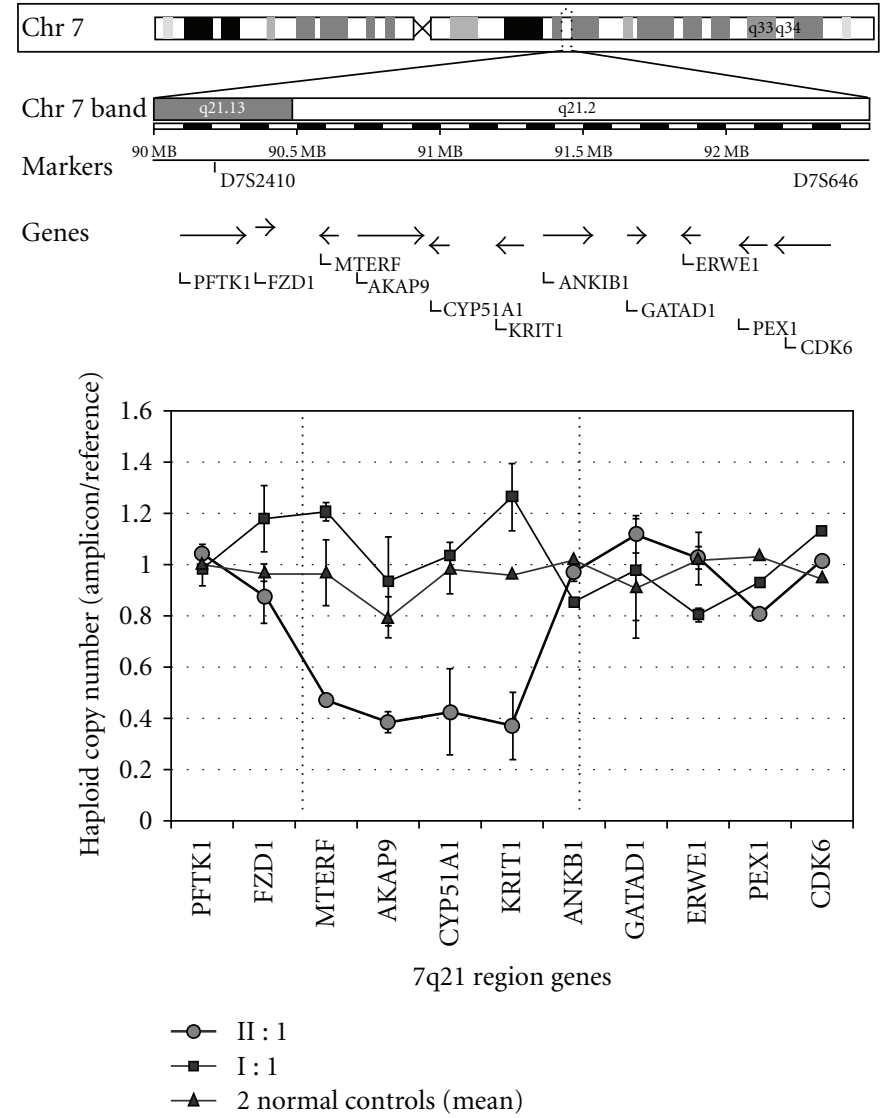

(b)

Figure 2: (a) Haplotype analysis of microsatellites markers from chromosome 7q21.1. The haplotype shared by the affected individuals (black-filled symbol) is "boxed". In the affected patients, the symbol (*) indicates the indefinite alleles, located in the deletion. Since the hemizygosity for the non shared alleles in the affected patients, the analysis, shows "homozygosity" for three markers (D7S2409, D7S1813, D7S1789), thus, the deleted alleles are indicated with the same symbol (*). (b) Measurements of copy number status ( \pm standard error mean) of genes mapping in the 7q21 chromosomal region determined by RT-QPCR. On the Top: the genomic organization of genes. Genes are shown above the horizontal axis, which also indicates the extension and orientation of each gene. Results from normal genomic DNA (2 normal controls mean), from the unaffected member of family (I: 1 sample), and in one affected members (II: 1$)$ are visualized by a closed triangle $(\boldsymbol{\Delta})$, a closed quare $(\boldsymbol{\square})$, and a closed circle $(\bullet)$, respectively. The genes between the two vertical gray dotted lines show a hemizigous deletion detectable by the copy number loss of the associated amplicons.

3.2.3. Gene Expression Analysis. To further confirm the inclusion of these genes into the genomic deletion, an expression analysis approach was set up for all genes located into the D7S2410 and D7S646 interval. FZD1 and PFTK1 were not analysed because these genes are not expressed in peripheral blood (data not shown). Results confirmed the decrease of expression of genes showing an $\mathrm{H}-\mathrm{CN}$ score of $0.5 \pm 0.2$ and a normal expression of genes with a D-CN of $1.0 \pm 0.2$ (Figure 3 ). The ANKIB1 gene was characterized by a discrepancy between the normal DNA D-CN (detected using a couple of primers located into the intron 14) and an evident decrease of expression level (detected using a couple of primers located into the exon 6).

\subsubsection{RT-QPCR Analysis for the ANKIB1 Gene Breakpoint} Determination. For the ANKIB1 gene, additional RT-QPCR analysis by using intragenic primers revealed that the deletion affected a large segment of the gene with a breakpoint located in the intron 10 flanked by the amplicons ANKIB1Ex11 (not deleted) and ANKIB1-Ex10 (deleted), (D-CN $=1.08 \pm 0.03$ versus $\mathrm{H}-\mathrm{CN}=0.53 \pm 0.02$, respectively, Figure 4).

\section{Discussion}

The use of MLPA in routine diagnostics increases dramatically the identification of large/small genomic deletion in human disease gene screening. Recently also for the three CCM associated genes, MLPA kits have been assessed and validated, and recent papers demonstrated the usefulness of this application and identified different partial or total deletions of these genes [11-15]. However, one limit of these technique, is the lack of the information about the heterozygosity of genomic region flanking the specific gene under study. This limit can be overcome by other techniques such as the RT-QPCR, or SNP Copy Number Variations 


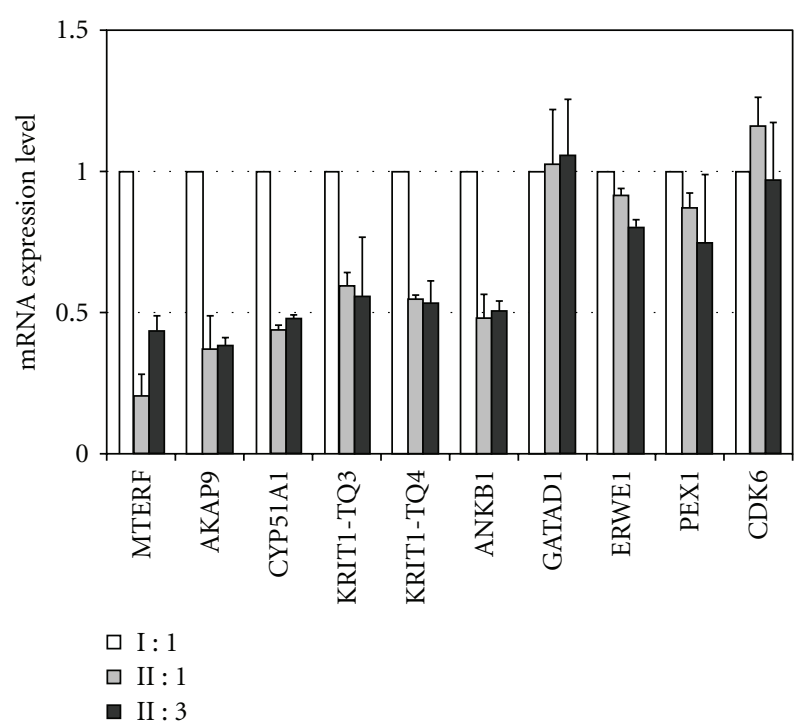

Figure 3: Copy number status Expression level analysis ( \pm standard error mean) of genes mapping in the 7q21 chromosomal region determined by RT-QPCR was determined by RT-QPCR. Average relative mRNA expression genes level from two affected members of CCM family (II : 1 and II : 3 ) related to the normal ones as calibrator ( $\mathrm{I}: 1)$ to $G A P D H$ gene as reference.

Assay. Although these techniques are more expensive and sometimes time consuming compared to the MLPA analysis, nevertheless, the assessment of the breakpoints allows to identify other possible deleted genes and to investigate their contribution to the disease.

Here we report a genomic deletion of about $700 \mathrm{~kb}$ across the CCM1 locus encompassing the MTERF, AKAP9, CYP51A1 genes, and $90 \%$ of the ANKIB1 gene. We wondered the possible relationship between the predicted function of the corresponding encoded proteins and the clinical features reported by our patients.

Few information about the function of ANKIB1 was available at the time of this work. though the ANKIB1 was recently described as a UIM (ubiquitin-interacting motif) protein with an ubiquitylation function [21]. Thus, at the moment, we are still unable to deduce if the haploinsufficiency of these proteins could influence the clinical phenotype of cavernous angiomas. The CYP51A1 gene encodes for the Sterol 14-alpha-demethylase, a member of the cytochrome P450 gene superfamily involved in sterol biosynthesis in fungi, plants, and animals [22]. There are no data about the possible involvement in human disease, and with the chemistry analysis performed on our patients.

The MTERF gene encodes the mitochondrial termination factor with a complex role in mitochondrial transcription arrest and transcription activation [23]. In particular, the MTERF protein binds the specific mtDNA region responsible for the MELAS syndrome, a mitochondrial disease characterized by mitochondrial myopathy, encephalopathy, lactic acidosis, and stroke-like episodes. The MELAS mutations reported commonly occur within the mtDNA binding site for the MTERF protein whose role is the termination of

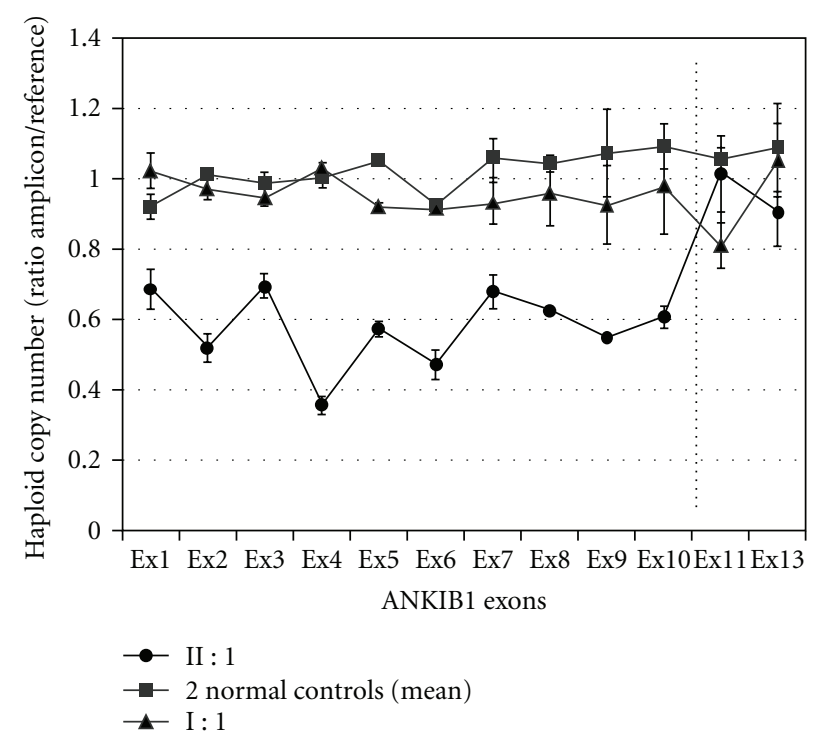

FIgURE 4: ANKIB1 gene breakpoint determination by RT-QPCR ( \pm standard error mean). Results from normal genomic DNA (2 normal controls mean), from the unaffected member of family (I: 1 sample), and in one affected members (II:1) are visualized by a closed triangle $(\boldsymbol{\Lambda})$, a closed square $(\boldsymbol{\square})$, and a closed circle $(\bullet)$, respectively. The ANKIB1 exons on the left of the vertical dotted lines (exon1-10) show the copy number loss of the associated amplicons.

transcription at the $16 \mathrm{~S}$ rRNA/tRNA (LeuUUR) gene boundary. One could ask if the stroke-like episodes and seizures, as part of symptoms showed by our patients, could overlap with those specific symptoms of MELAS diseases, rather than exclusively due to the KRIT1 gene haploinsufficiency. Unfortunately, there are no data supporting the assumption that the MELAS syndrome could be caused by the loss of expression of the MTERF protein as well as no relationship was reported between these CCM and MELAS. Thus, this hypothesis needs to be confirmed by wider epidemiological studies.

The AKAP9 gene encodes for the A-kinase anchor protein-9, a scaffolding protein that determines the subcellular localization of protein kinase A and enzymes that regulate the PKA pathway and the $\mathrm{I}(\mathrm{Ks})$ potassium channel in the heart [24]. A recent functional study described a link between genetic perturbations in AKAP9 and congenital long QT syndrome (LQTS), electrocardiographically characterized by a prolonged QT interval and polymorphic ventricular arrhythmias which may result in recurrent syncope, seizures, or sudden death [25]. However, no alteration in ECGs was observed in our CCM affected patients that could be related to this syndrome.

One notable clinical feature in our family was the anticipation of the symptoms. Although such anticipation was already described, it does not represent a common feature of the CCM and molecular mechanism remains unknown $[26,27]$. It is possible that in our family the anticipation could depend on other genetic factors, mainly associated with the intrinsic genomic variability that leads the mutation 
carriers to be more or less affected by haploinsufficiency of the three CCM-associated genes.

In conclusion, we report the molecular characterization of an interstitial small genomic deletion of the 7q21.2 region in a CCMs affected family, encompassing the KRIT1 gene. This paper focuses on the utility of a fine molecular characterization of this type of genomic rearrangement in CCM affected families to investigate a possible role of the flanking deleted genes. However, taking into account the absence in our survey of any other clinical features in addition to the multiple cavernous angiomas, our results can only confirm the loss of function mechanism for the already known CCM1 locus. Further evaluations of clinical features during the follow-up of our patients could contribute to clarify this issue.

\section{Acknowledgments}

The authors acknowledge the families participating to this study. they thank Dr. Silvana Muscarella for the English revision of the paper. This work was supported by Italian Ministry of Health (Ricerca Corrente 2004, 2005).

\section{Competing Interests}

The authors declare that they have no financial or personal relationships with other people or organizations that could inappropriately influence or bias this work.

\section{References}

[1] D. Rigamonti, M. N. Hadley, B. P. Drayer et al., "Cerebral cavernous malformation. Incidence and familial occurrence," New England Journal of Medicine, vol. 319, no. 6, pp. 343-347, 1988.

[2] D. S. Russel and L. J. Rubinstein, Pathology of Tumors of the Nervous System, Williams and Wilkins, Baltimore, Md, USA, 5th edition, 1989.

[3] P. Labauge, S. Laberge, L. Brunereau, C. Levy, and E. TournierLasserve, "Hereditary cerebral cavernous angiomas: clinical and genetic features in 57 French families Societe Francaise de Neurochirurgie," Lancet, vol. 352, pp. 1892-1897, 1998.

[4] P. Otten, G. P. Pizzolato, B. Rilliet, and J. Berney, "A study of 131 cases of cavernomas of the CNS, discovered on retrospective analysis of 24,535 autopsies," Neurochirurgie, vol. 35, no. 2, pp. 82-83, 1989.

[5] H. D. Craig, M. Günel, O. Cepeda et al., "Multilocus linkage identifies two new loci for a mendelian form of stroke, cerebral cavernous malformation, at 7p15-13 and 3q25.2-27," Human Molecular Genetics, vol. 7, no. 12, pp. 1851-1858, 1998.

[6] C. Denier, P. Labauge, L. Brunereau et al., "Clinical features of cerebral cavernous malformations patients with KRIT1 mutations," Annals of Neurology, vol. 55, no. 2, pp. 213-220, 2004.

[7] S. Laberge-Le Couteulx, H. H. Jung, P. Labauge et al., "Truncating mutations in CCM1, encoding KRIT1, cause hereditary cavernous angiomas," Nature Genetics, vol. 23, no. 2, pp. 189-193, 1999.
[8] C. L. Liquori, M. J. Berg, A. M. Siegel et al., "Mutations in a gene encoding a novel protein containing a phosphotyrosinebinding domain cause type 2 cerebral cavernous malformations," American Journal of Human Genetics, vol. 73, no. 6, pp. 1459-1464, 2003.

[9] F. Bergametti, C. Denier, P. Labauge et al., "Mutations within the programmed cell death 10 gene cause cerebral cavernous malformations," American Journal of Human Genetics, vol. 76, no. 1, pp. 42-51, 2005.

[10] D. J. Verlaan, J. Roussel, S. B. Laurent, C. E. Elger, A. M. Siegel, and G. A. Rouleau, "CCM3 mutations are uncommon in cerebral cavernous malformations," Neurology, vol. 65, no. 12, pp. 1982-1983, 2005.

[11] C. L. Liquori, M. J. Berg, F. Squitieri et al., "Deletions in CCM2 are a common cause of cerebral cavernous malformations," American Journal of Human Genetics, vol. 80, no. 1, pp. 69-75, 2007.

[12] C. L. Liquori, S. Penco, J. Gault et al., "Different spectra of genomic deletions within the CCM genes between Italian and American CCM patient cohorts," Neurogenetics, vol. 9, no. 1, pp. 25-31, 2008.

[13] S. Gaetzner, S. Stahl, O. Sürücü et al., "CCM1 gene deletion identified by MLPA in cerebral cavernous malformation," Neurosurgical Review, vol. 30, no. 2, pp. 155-159, 2007.

[14] U. Felbor, S. Gaetzner, D. J. Verlaan, R. Vijzelaar, G. A. Rouleau, and A. M. Siegel, "Large germline deletions and duplication in isolated cerebral cavernous malformation patients," Neurogenetics, vol. 8, no. 2, pp. 149-153, 2007.

[15] S. Penco, R. Ratti, E. Bianchi et al., "Molecular screening test in familial forms of cerebral cavernous malformation: the impact of the Multiplex Ligation-dependent Probe Amplification approach. Laboratory investigation," Journal of Neurosurgery, vol. 110, no. 5, pp. 929-934, 2009.

[16] J. Sambrook, E. F. Fritsch, and T. Maniatis, Molecular Cloning: A Laboratory Manual, Cold Spring Harbor Laboratory, New York, NY, USA, 1989.

[17] V. Guarnieri, L. A. Muscarella, R. Amoroso et al., "Identification of two novel mutations and of a novel critical region in the KRIT1 gene," Neurogenetics, vol. 8, no. 1, pp. 29-37, 2007.

[18] K. J. Livak and T. D. Schmittgen, "Analysis of relative gene expression data using real-time quantitative PCR and the $2^{-\triangle \Delta C T}$ method," Methods, vol. 25, no. 4, pp. 402-408, 2001.

[19] J. Vandesompele, K. De Preter, F. Pattyn et al., "Accurate normalization of real-time quantitative RT-PCR data by geometric averaging of multiple internal control genes," Genome biology, vol. 3, no. 7, research0034, pp. 1-11, 2002.

[20] J. Hoebeeck, R. van der Luijt, B. Poppe et al., "Rapid detection of VHL exon deletions using real-time quantitative PCR," Laboratory Investigation, vol. 85, no. 1, pp. 24-33, 2005.

[21] S. L. H. Miller, E. Malotky, and J. P. O'Bryan, “Analysis of the role of ubiquitin-interacting motifs in ubiquitin binding and ubiquitylation," Journal of Biological Chemistry, vol. 279, no. 32, pp. 33528-33537, 2004.

[22] H. Vanden Bossche, F. Dromer, I. Improvisi, M. LozanoChiu, J. H. Rex, and D. Sanglard, "Antifungal drug resistance in pathogenic fungi," Medical Mycology, vol. 36, no. 1, Supplement, pp. 119-128, 1998.

[23] M. Roberti, P. L. Polosa, F. Bruni et al., "The MTERF family proteins: mitochondrial transcription regulators and beyond," Biochimica et Biophysica Acta, vol. 1787, no. 5, pp. 303-311, 2009.

[24] G. McConnachie, L. K. Langeberg, and J. D. Scott, "AKAP signaling complexes: getting to the heart of the matter," Trends in Molecular Medicine, vol. 12, no. 7, pp. 317-323, 2006. 
[25] L. Chen, M. L. Marquardt, D. J. Tester, K. J. Sampson, M. J. Ackerman, and R. S. Kass, "Mutation of an A-kinaseanchoring protein causes long-QT syndrome," Proceedings of the National Academy of Sciences of the United States of America, vol. 104, no. 52, pp. 20990-20995, 2007.

[26] A. M. Siegel, E. Andermann, A. Badhwar et al., "Anticipation in familial cavernous angioma: a study of 52 families from international familial cavernous angioma study," Lancet, vol. 352, no. 9141, pp. 1676-1677, 1998.

[27] A. M. Siegel, F. Andermann, A. Badhwar et al., "Anticipation in familial cavernous angioma: ascertainment bias orgenetic cause," Acta Neurologica Scandinavica, vol. 98, no. 6, pp. 372376, 1998. 

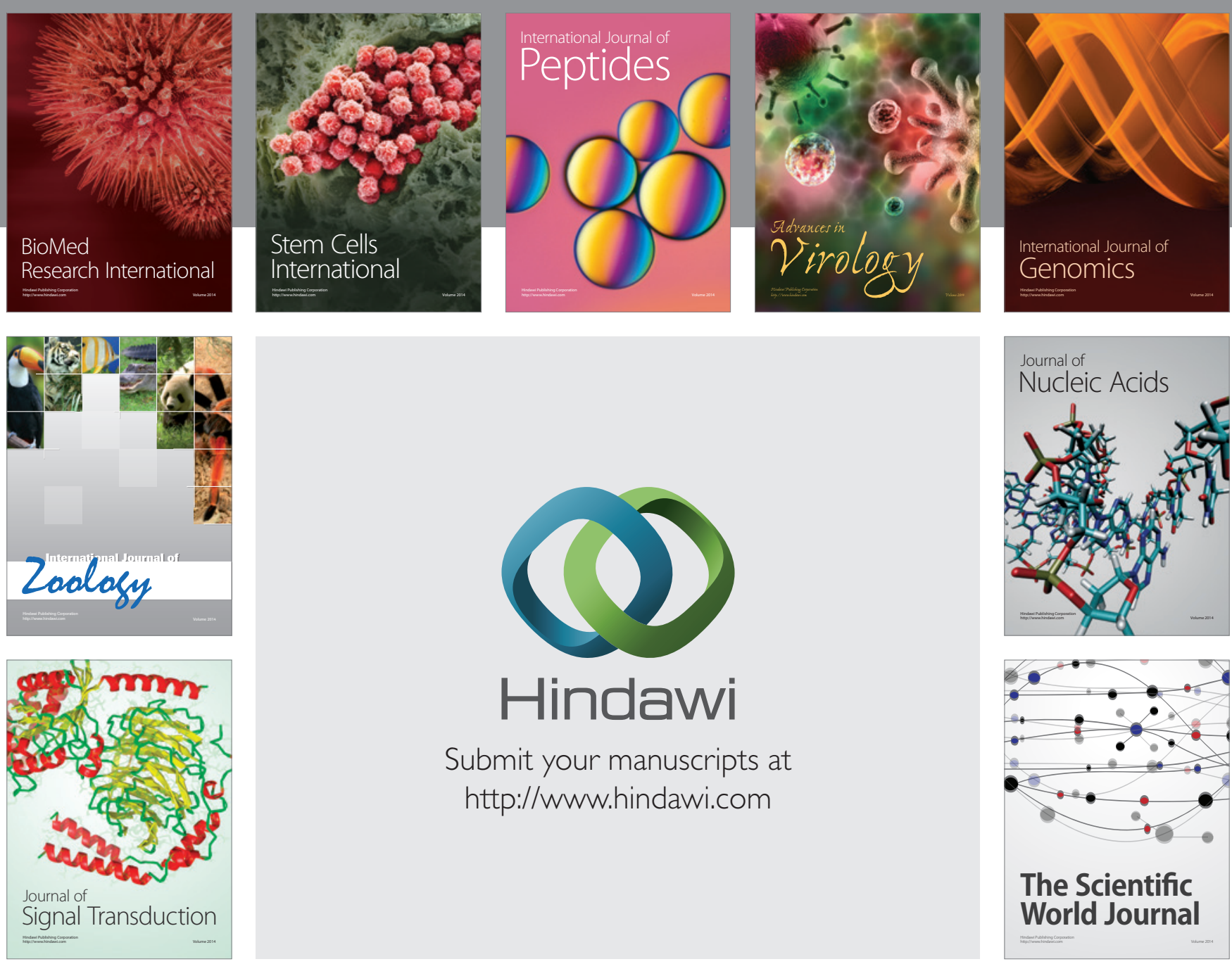

Submit your manuscripts at

http://www.hindawi.com
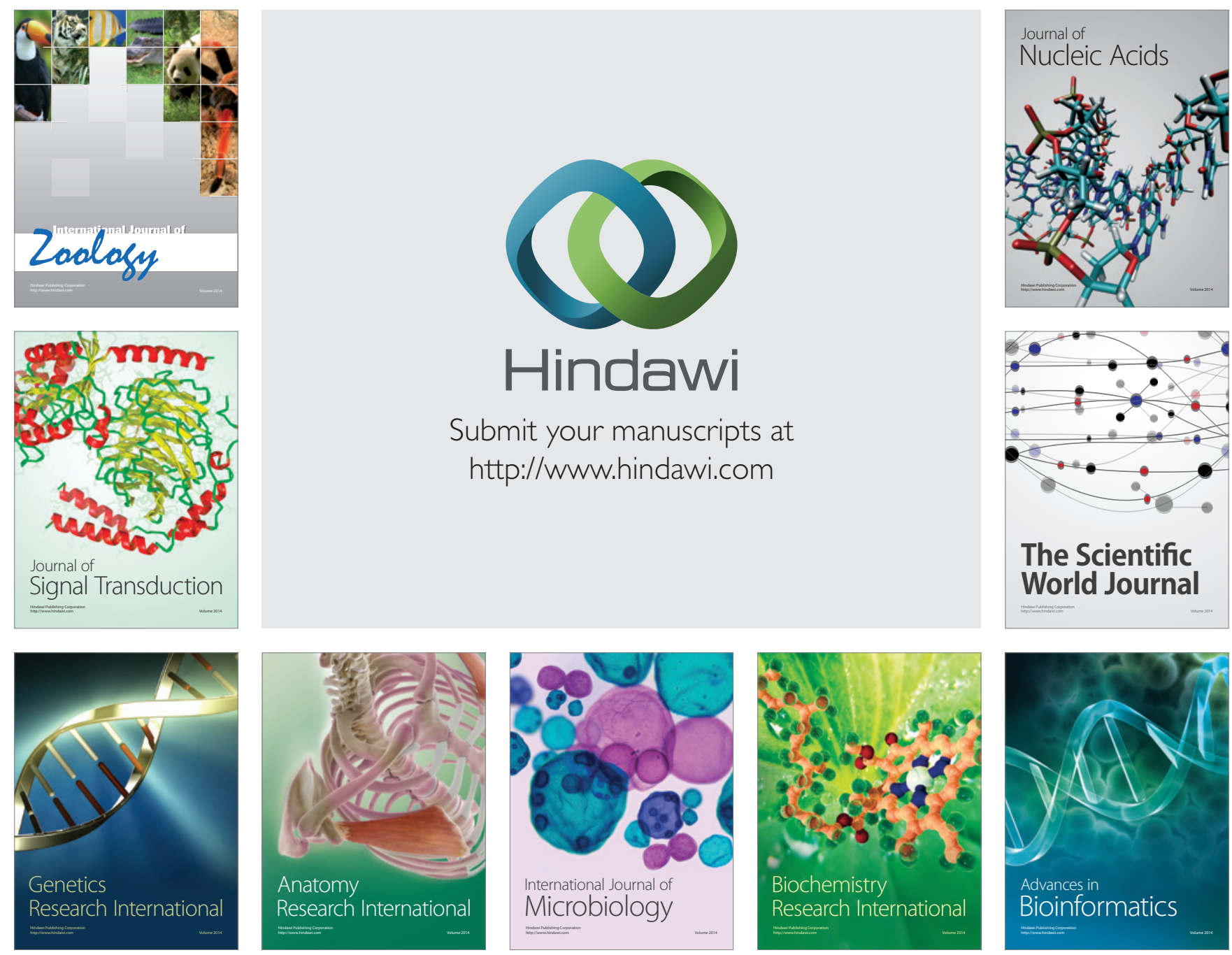

The Scientific World Journal
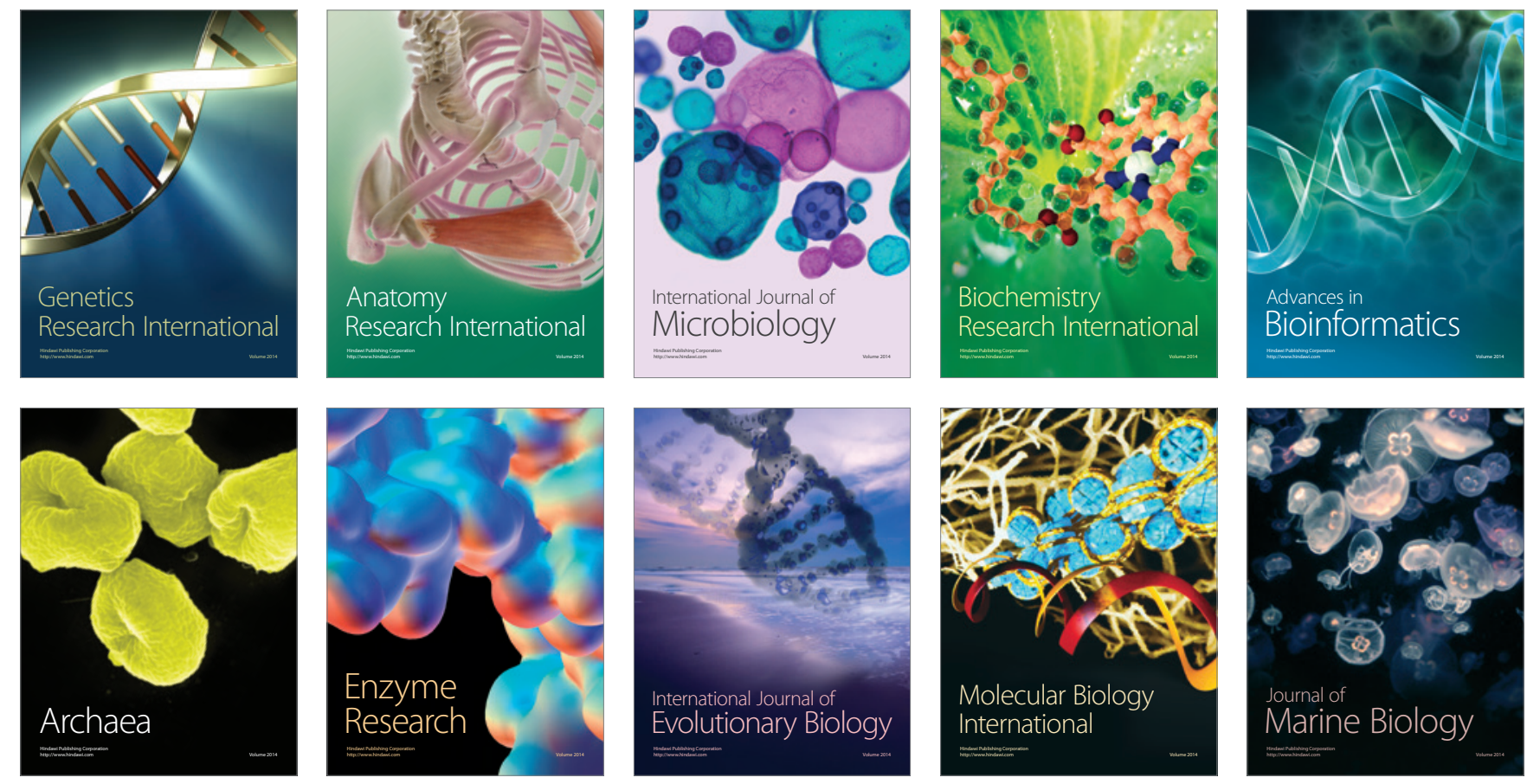